\title{
Clinical research on alternating hyperfraction radiotherapy for massive hepatocellular carcinoma
}

\author{
ZHIXIONG LONG ${ }^{1 *}$, BIN WANG $^{1 *}$, DAN TAO $^{2 *}$, YANPING LIU ${ }^{2}$, JIANGZHOU ZHANG $^{2}$, \\ JIAAN TAN ${ }^{3}$, JING LUO $^{3}$, FEIFEI SHI ${ }^{3}$ and ZEZHANG TAO ${ }^{1}$ \\ ${ }^{1}$ Department of Otolaryngology - Head and Neck Surgery, Renmin Hospital of Wuhan University, \\ Wuhan, Hubei 430060; ${ }^{2}$ Department of Oncology, The Fifth Hospital of Wuhan, Wuhan, Hubei 430050; \\ ${ }^{3}$ Department of Oncology, Hubei Provincial Traditional Chinese Medical Hospital, Wuhan, Hubei 430061, P.R. China
}

Received August 2, 2014; Accepted April 13, 2015

DOI: $10.3892 / 01.2015 .3185$

\begin{abstract}
The delivery of high tumoricidal doses of radiation with low rates of toxicity is of particular significance for massive hepatocellular carcinoma (HCC) radiotherapy. In order to observe the efficacy and adverse reactions of alternating hyperfraction radiotherapy treatment of massive $\mathrm{HCC}$, seventy-two cases of massive HCC were randomly divided into two groups, group A and group B. The liver lesions of group A were divided into sublesions and treated with alternating hyperfraction radiotherapy [intensity modulated radiotherapy (IMRT)]. The interval between radiotherapy to the sublesions was a minimum of six hours. The average radiotherapy dose to the sublesions was $2 \mathrm{~Gy} /$ fraction, once a day, five times per week, treating the gross tumor volume with a total dose of 40-50 Gy, and the clinical target volume with a total dose of 30-40 Gy. By contrast, the lesions of group B were not divided into sublesions for the IMRT treatment, but were treated with an otherwise identical protocol, by 2 Gy/fraction, once a day, five times per week, and with the same total dose. Patients were followed up with regular blood tests, liver function tests, measurements of serum $\alpha$-fetoprotein levels and contrast-enhanced magnetic resonance imaging (MRI) of the liver. Treatment responses were assessed every 3 months by MRI. The results revealed that the overall response rates of the two groups were 82.9 and $81.3 \%$, respectively $(\mathrm{P}=0.864)$. The alternating hyperfraction radiotherapy protocol resulted in enhanced survival $(\mathrm{P}=0.002)$. The median survival time of the two groups was 9.7 and 6.5 months, respectively. The
\end{abstract}

Correspondence to: Dr Zezhang Tao, Department of Otolaryngology - Head and Neck Surgery, Renmin Hospital of Wuhan University, 99 Zhangzhidong Road, Wuhan, Hubei 430060, P.R. China

E-mail: tdjgs2008@126.com

*Contributed equally

Key words: alternating hyperfraction radiotherapy, massive hepatocellular carcinoma, radiation-induced liver disease overall 6-month, 1-year, 2-year and 3-year survival rates of the two groups were 62.9 and $59.4 \%(\mathrm{P}=0.770), 48.6$ and $21.9 \%(\mathrm{P}=0.040), 17.1$ and $0.0 \%(\mathrm{P}=0.025)$ and 2.9 and $0.0 \%$ $(\mathrm{P}=1.000)$, respectively. The I-II degree of abnormal liver function and radiation-induced liver disease of group $\mathrm{B}$ was higher than that of group A ( $\mathrm{P}=0.021$ and 0.046 , respectively). In addition, the incidence rate of radiation-induced liver injury of group A was lower than that of group B. Therefore, treatment of massive HCC with alternating hyperfraction radiotherapy improved the quality of life and prolonged the overall survival time, compared with conventional IMRT, suggesting that it was an effective radiation pattern.

\section{Introduction}

Primary hepatocellular carcinoma (HCC) is the third most common cause of cancer-associated death worldwide, with $85 \%$ of cases occurring in developing countries. Primary HCC has also become the fastest growing cause of cancer-associated death in the United States of America $(1,2)$. HCC is classified into four subtypes: Massive, nodular, diffuse and small HCC, among which the massive type ( $\geq 10 \mathrm{~cm})$ is most common (3). Surgery is the curative modality of treatment for patients presenting with a solitary lesion without vascular invasion, and with sufficient underlying liver function (4). However, due to the large volume of massive HCC lesions, major blood vessels, including the portal vein, hepatic artery and vena cava are frequently invaded (5). In addition, the majority of patients with HCC suffer from cirrhosis or abnormal liver function, which may also result in difficulties for surgical intervention (6). Thus, in the majority of massive HCC cases, surgery is unfeasible due to the disease burden or location, comorbidities or inadequate functional liver reserve (4).

Despite the fact that liver tumors are sensitive to the effects of radiation, traditional radiotherapy has not had a significant role in the treatment of HCC. This is primarily due to the challenges associated with the delivery of a sufficient dose of radiation to the target to be tumoricidal, without inducing significant toxicity (7). Following the development of modern stereotactic radiotherapy techniques, the safe delivery of radiation to the liver has become more achievable, and radiotherapy has had a growing role in the local treatment of $\operatorname{HCC}(6,8,9)$. 
However, due to the large volume of massive HCC lesions and the poor radiation tolerance of normal liver tissue, increasing the radiation dose has remained an issue and thus the curative effect of radiotherapy is limited (10). Therefore, the delivery of higher tumoricidal radiation doses to focal HCC, with low rates of toxicity is a desirable development to improve existing treatments (11). In the present study, alternating hyperfraction radiotherapy was compared with regular intensity modulated radiotherapy (IMRT) treatment in the treatment of patients with massive HCC. The results may provide evidence for the development of a novel radiotherapy treatment strategy for massive HCC.

\section{Materials and methods}

Patient characteristics. The present prospective study of alternating hyperfraction radiotherapy for patients with massive HCCs was approved by the ethics committee of the Fifth Hospital of Wuhan (Wuhan, Hubei, China) and was initiated in May 2008. Written informed consent was obtained from the patients for participation in the study. Massive HCCs were defined as those with a maximum tumor diameter of $\geq 10 \mathrm{~cm}$ (12-14). The 72 patients, recruited between May 2008 and June 2011 at the Fifth Hospital of Wuhan, comprised 40 males and 32 females, with an age range of 38-65 years, and a mean age of 54.2 years. The tumor sizes, determined using contrast-enhanced magnetic resonance imaging (MRI; Achieva 1.5T, Philips Medical Systems, Best, Netherlands) by the radiologist, were $10-20 \mathrm{~cm}$ in diameter. The eligibility criteria for patients were: Karnofsky performance status score, $\geq 70$ (15); Child-Pugh class, A or B (16); serum glutamic pyruvic transaminase, $<100 \mathrm{U} / \mathrm{l}$; serum glutamic-oxaloacetic aminotransferase, $<100 \mathrm{U} / 1$; serum total bilirubin, $<170 \mu \mathrm{mol} / \mathrm{l}$; ultrasonic examination without massive ascites and an absence of intrahepatic metastasis or other distant metastasis.

Radiotherapy equipment. An Elekta electron linear precise accelerator [configuration iView-GT portal imaging verification system, 40 pairs of electric MLC; Elekta AB (Publ), Stockholm, Sweden], Xinhua SL-11 simulator (Shandong Xinhua Medical Instrument Co., Ltd, Shandong, China), Philips 64-slice spiral computed tomography scanner (Brilliance 64, Philips Medical Systems), Elekta Precise three-dimensional (3D) treatment planning system [Elekta AB (Publ)], immobilization vacuum pad (Shanghai Gerui Co., Ltd, Shanghai, China) and an abdominal bandage (Hengshui Runde Medical Instrument Co., Ltd, Hebei, China) were used in the present study.

Radiotherapy treatment protocol. The patients were randomly divided into two groups $(n=36)$ : group A and group B. For the alternating hyperfractionated intensity modulated conformal radiotherapy treatment (group A), the liver lesion was divided into two sublesions, gross tumor volume (GTV)1 and GTV2, based on the anatomical features of the tumor. The interval between the radiotherapy treatment of the GTV1 and GTV2 sublesions was $\geq 6 \mathrm{~h}$, to avoid radiation hot spots. The average radiotherapy dose of the sublesions was 2 Gy/fraction, once a day, five times per week for 4-5 weeks. The GTV received a total dose of 40-50 Gy, and the clinical target volume (CTV) received a total dose of 30-40 Gy. For the regular intensity modulated conformal radiotherapy group (group B), the liver lesions were treated with intensity modulated radiotherapy (IMRT) at a dose of $2 \mathrm{~Gy} /$ fraction, once a day, five times per week for 4-5 weeks. The GTV was treated with a total dose of 40-50 Gy, and the CTV received a total dose of 30-40 Gy. Radiotherapy was performed with the aforementioned Elekta electron linear accelerator and precise treatment planning system. The abdominal bandage was used to control breathing during radiotherapy, in order to reduce tumor motion. Target volume delineation was performed by the same doctor and medical physicist for all patients. To perform palliative radiotherapy, an electronic image treatment verification system [Elekta AB (Publ)] was used to improve the precision. Based on the CTV, the crown sagittal axis of the planning target area (PTV) was extended outwards by $3 \mathrm{~mm}$ and the long axis of the body was extended outwards by $5 \mathrm{~mm}$. In total, 7-9 irradiation fields were designed, and $85-90 \%$ of the isodose curve covered the PTV. Protection was provided for the normal liver and adjacent organs (stomach, duodenum, pancreas, kidneys) as much as possible, using the Elekta Precise 3D treatment planning system. During radiotherapy, the patients were treated with liver protection drugs glutathione (1.2-1.8 g/day) and magnesium isoglycyrrhizinate (0.1-0.3 g/day). Regular inspections of peripheral blood and liver function were conducted during the course of treatment.

Follow up. Patients were monitored over the 2-3 months following treatment, and trimonthly thereafter. Regular blood tests, liver function tests, measurements of serum $\alpha$-fetoprotein (AFP) and contrast-enhanced MRI (Achieva 1.5T, Philips Medical Systems) of the liver were performed at every follow-up appointment. Treatment responses were evaluated by MRI every 3 months, using the modified Response Evaluation Criteria in Solid Tumors (17). The treatment responses were defined as follows: Complete regression (CR), total disappearance of the tumor; partial regression (PR), a decrease of $>50 \%$ of the tumor size; stable disease (SD), a decrease of $<50 \%$ of the tumor or no change in tumor volume; progressive disease (PD), tumor progression (18). Adverse reactions to radiotherapy were evaluated by the standard criteria of the American Radiation Therapy Oncology Group (19). Survival was evaluated from the date of commencement of the treatment (20). The follow-up was completed in June 2014.

Statistics analysis. SPSS 17.0 statistical software (SPSS, Inc., Chicago, IL, USA) was used for statistical analysis. Effective rates, survival rates and the incidence of adverse reactions were analyzed using the $\chi^{2}$ test. The Kaplan-Meier method was used to estimate survival rates, and the log-rank test was used to compare differences in survival. A two-sided $\mathrm{P}<0.05$ was considered to indicate a statistically significant difference.

\section{Results}

Treatment outcomes. One patient in group A was unable to complete the radiotherapy treatment due to upper gastrointestinal hemorrhage. Four patients in group B did not complete the radiotherapy course as a result of severe reactions. The follow-up rate was $100 \%$ amongst the remaining patients. The 
Table I. The changes in serum AFP of groups A $(n=35)$ and B $(n=32)$ to radiotherapy.

\begin{tabular}{llll}
\hline & \multicolumn{2}{c}{ Serum AFP (mean \pm SD), $\mu \mathrm{g} / 1$} & \\
\cline { 2 - 3 } Group & Before treatment & After treatment & $<0.0001$ \\
\hline A & $557.78 \pm 142.08$ & $286.62 \pm 114.81$ & $<0.0001$ \\
\hline
\end{tabular}

Group A, alternating hyperfraction radiotherapy; Group B, regular intensity modulated radiotherapy. AFP, $\alpha$-fetoprotein; SD, standard deviation.

Table II. Treatment responses of groups A $(n=35)$ and B $(n=32)$ to radiotherapy.

\begin{tabular}{lcc}
\hline & \multicolumn{2}{c}{ Patients, \% (n) } \\
\cline { 2 - 3 } Treatment response & Group A & Group B \\
\hline CR & $5.7(2)$ & $3.1(1)$ \\
PR & $28.6(10)$ & $21.9(7)$ \\
SD & $48.6(17)$ & $18.7(6)$ \\
PD & $17.1(6)$ & $81.3(26)$ \\
CR+PR+SD & $82.9(29)$ & 0.864 \\
\hline
\end{tabular}

Group A, alternating hyperfraction radiotherapy; Group B, regular intensity modulated radiotherapy. CR, complete response; PR, partial response; $\mathrm{SD}$, stable disease; $\mathrm{PD}$, progressive disease.

Table III. Adverse reactions of groups A $(n=35)$ and B $(n=32)$ to radiotherapy.

\begin{tabular}{lccc}
\hline Adverse reaction $^{\text {a }}$ & Group A, \% (n) & Group B, \% (n) & P-values \\
\hline I-II degree gastrointestinal reactions & $82.9(29)$ & $81.3(26)$ & $87.5(28)$ \\
I-II degree abnormal liver function & $62.9(22)$ & $71.9(23)$ & 0.864 \\
I-II degree myelosuppression & $65.7(23)$ & $31.3(10)$ & 0.021 \\
Radiation-induced liver disease & $11.4(4)$ & 0.046 \\
\hline
\end{tabular}

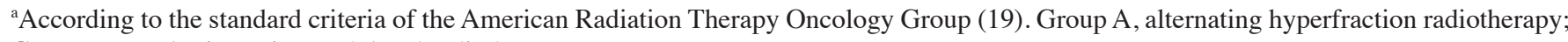
Group B, regular intensity modulated radiotherapy.

results of the follow-up 2-3 months subsequent to treatment revealed that the average values of serum AFP for groups A and $\mathrm{B}$ were $286.62 \pm 114.81$ and $299.67 \pm 115.03 \mu \mathrm{g} / 1$, respectively, compared with $557.78 \pm 142.08$ and $547.02 \pm 151.01 \mu \mathrm{g} / \mathrm{l}$, respectively, prior to treatment. The changes in serum AFP of the two groups were statistically significant $(\mathrm{P}=0.000$; Table I). The total effective rate of treatment was $82.9 \%(29 / 35)$ in group $\mathrm{A}$, and the $\mathrm{CR}, \mathrm{PR}, \mathrm{SD}$ and $\mathrm{PD}$ rates were $5.7(2 / 35)$, $28.6(10 / 35), 48.6(17 / 35)$ and $17.1 \%$ (6/35), respectively. In group B, the total effective rate was $81.3 \%(26 / 32)$, and the CR, PR, SD and PD rates were 3.1 (1/32), 21.9 (7/32), 56.3 (18/32) and $18.7 \%(6 / 32)$, respectively. The overall response rates of the two groups were equivalent $(\mathrm{P}=0.864$; Table II).

The follow-up ended in June 2014. The median survival time of patients in group A was 9.7 months, compared with 6.5 months in those of group B. The survival time of group A was significantly longer than that of group $\mathrm{B}(\mathrm{P}=0.002$; Fig. 1).

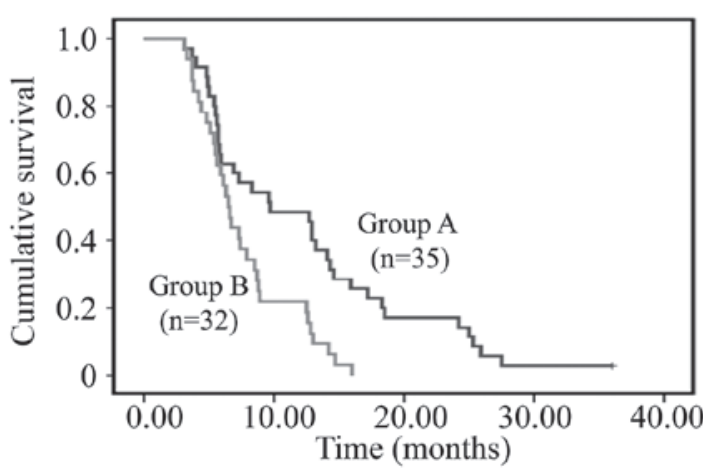

Figure 1. Survival times of patients in group A are longer than those of group B. Kaplan-Meier curves of survival durations of patients with massive HCC following alternating hyperfraction radiotherapy (group A) and regular IMRT (group B). Survival durations were significantly longer in patients receiving alternating hyperfraction radiotherapy (median survival, 9.7 months) than those receiving regular IMRT radiotherapy (median survival, 6.5 months; $\mathrm{P}=0.002$ ). IMRT, intensity modulated radiotherapy. 
The 6-month, 1-year, 2-year and 3-year overall survival rates of the two groups were 62.9 and $59.4 \%(\mathrm{P}=0.770), 48.6$ and $21.9 \%(\mathrm{P}=0.040), 17.1$ and $0.0 \%(\mathrm{P}=0.025)$ and 2.9 and $0.0 \%$ $(\mathrm{P}=1.000)$, respectively.

Treatment-associated toxicity. The major toxic effects of radiotherapy during the treatment were gastrointestinal reactions, abnormal liver function and myelosuppression. The I-II degree gastrointestinal reactions and I-II degree myelosuppression of the two groups were similar (Table III). However, the I-II degree of abnormal liver function and radiation-induced liver disease (RILD) of group B were significantly higher than that of group A ( $\mathrm{P}=0.021$ and 0.046 , respectively; Tables I and III). RILD was determined by an elevation of alkaline phosphatase level of $\geq 2$-fold and/or elevated transaminases of $\geq 4$-fold the upper limit of normal levels (18).

\section{Discussion}

HCC is one of the most common types of cancer worldwide, and it is frequently not diagnosed until an advanced stage when the majority of potentially curative therapies, for example resection, transplantation or transarterial interventions, are of limited efficacy $(21,22)$. Previously, due to the low tolerance of normal liver tissues to radiation, the use of radiotherapy for the treatment of HCC has been limited (23). Following the development of modern computers and medical imaging technology, radiation therapy of tumors has entered a novel era of 3D conformal radiotherapy (3D-CRT). 3D-CRT ensures that the target receives identical high-dose conformal irradiation, so that the radiation dose to the surrounding normal tissue is markedly reduced, thereby generating the conditions for the incremental target area (24). It was demonstrated that there was a dose-response association in local radiotherapy for primary HCC, and thus 3D-CRT may potentially be used for the treatment of primary $\mathrm{HCC}(25,26)$. In addition, stereotactic body radiotherapy has emerged as a viable treatment option for patients with liver tumors unsuitable for surgery, liver transplantation, or radiofrequency ablation (20).

Recently, radiotherapy technology has evolved from 3D-CRT to a more advanced form, termed IMRT, which facilitates the application of a substantial dose of radiation to a tumor whilst avoiding damage to local radiosensitive organs (27). IMRT may enhance the quality of radiation plans by utilizing an inverse planning algorithm to generate complex spatial dose distributions and, therefore, conform more closely to the target volume (27). It was demonstrated that IMRT may be an effective treatment for locally advanced HCC, which provides survival benefit without increasing severe toxicity (25). IMRT achieved a significantly higher conformal index and lower hot spot values than those of 3D-CRT (26). However, it was reported that, for tumors of diameter $>8 \mathrm{~cm}$, the value of mean dose for 3D-CRT was lower than that of IMRT, indicating that 3D-CRT was a more suitable strategy for the treatment of larger tumors (28). For massive HCC, a larger volume of normal liver is irradiated, and thus the majority of patients suffer from cirrhosis and abnormal liver function. This makes it more difficult to deliver high-dose radiation to the localized tumor area without significantly damaging the surrounding normal liver tissue. Thus, dose and volume reduction for the normal liver is required in order to improve the effects of radiotherapy for massive HCC. In addition, $\mathrm{HCC}$ is an early response tissue, whereas normal liver is a late response tissue (29). According to the principle of radiobiology, in order to reduce the radiation damage to the normal liver, the split dose should generally be $\leq 2$ Gy (30).

In the present study, the massive liver lesions of group A were divided into sublesions, GTV1 and GTV2, and alternating hyperfraction IMRT was designed to treat massive HCC. The interval of the radiotherapy to the sublesions was $\geq 6 \mathrm{~h}$. The average radiotherapy dose to the sublesions was $2 \mathrm{~Gy} /$ fraction, once a day, five times per week, with a total dose of 30-40 Gy. The results revealed that the alternating hyperfraction radiotherapy treatment strategy resulted in enhanced survival rates and reduced risk of I-II degree of abnormal liver function and RILD. The alternating hyperfraction IMRT protocol may alleviate the overall damage to the normal liver by reducing the radiation exposure and providing a greater period of time for repair, whilst ensuring the successful completion of radiotherapy for the tumor. In addition, an abdominal bandage was used to control breathing during radiotherapy in order to reduce tumor motion, thereby circumventing additional radiation-induced normal liver injury. Therefore, alternating hyperfraction provides an improved radiation pattern for the treatment of massive HCC. RILD is the most severe complication induced by radiotherapy, and may result in hepatic failure and death. Furthermore, it has been demonstrated that HCC may be more safely treated with spot-scanning proton therapy, in order to reduce the risk of RILD, particularly if the nominal tumor diameter is $>6.3 \mathrm{~cm}$ (31). Therefore, this alternating hyperfraction radiotherapy method may offer more effective outcomes for the treatment of HCC in the future.

In conclusion, the present study revealed that, compared with intensity modulated radiotherapy, the use of alternating hyperfraction radiotherapy may reduce the incidence rate of radiation-induced liver injury, improve patient quality of life and prolong the survival time, suggesting that it is an effective radiation pattern for the treatment of massive HCC.

\section{Acknowledgements}

The present study was supported by the Wuhan Public Health Bureau, China (grant no. WX13C36).

\section{References}

1. Center MM and Jemal A: International trends in liver cancer incidence rates. Cancer Epidemiol Biomarkers Prev 20: 2362-2368, 2011

2. Jemal A, Bray F, Center MM, Ferlay J, Ward E and Forman D: Global cancer statistics. CA Cancer J Clin 61: 69-90, 2011.

3. Willatt JM, Hussain HK, Adusumilli S and Marrero JA: MR Imaging of hepatocellular carcinoma in the cirrhotic liver: Challenges and controversies. Radiology 247: 311-330, 2008.

4. Aitken KL and Hawkins MA: The role of radiotherapy and chemoradiation in the management of primary liver tumours. Clin Oncol (R Coll Radiol) 26: 569-580, 2014.

5. Vauthey JN, Lauwers GY, Esnaola NF, Do KA, Belghiti J, Mirza N, et al: Simplified staging for hepatocellular carcinoma. J Clin Oncol 20: 1527-1536, 2002 .

6. Klein J and Dawson LA: Hepatocellular carcinoma radiation therapy: Review of evidence and future opportunities. Int $\mathbf{J}$ Radiat Oncol Biol Phys 87: 22-32, 2013.

7. Forner A, Llovet JM and Bruix J: Hepatocellular carcinoma. Lancet 379: 1245-1255, 2012. 
8. Lock MI, Hoyer M, Bydder SA, et al: An international survey on liver metastases radiotherapy. Acta Oncol 51: 568-574, 2012.

9. Culleton S, Jiang H, Haddad CR, et al: Outcomes following definitive stereotactic body radiotherapy for patients with Child-Pugh B or C hepatocellular carcinoma. Radiother Oncol 111: 412-417, 2014.

10. Zeng ZC, Tang ZY, Fan J, et al: A comparison of chemoembolization combination with and without radiotherapy for unresectable hepatocellular carcinoma. Cancer J 10: 307-316, 2004

11. Park HC, Seong J, Tanaka M, et al: Multidisciplinary management of nonresectable hepatocellular carcinoma. Oncology 81 (Suppl 1): S134-S140, 2011.

12. Carr BI and Guerra V: Features of massive hepatocellular carcinomas. Eur J Gastroenterol Hepatol 26: 101-108, 2014.

13. Carr BI, Guerra V and Pancoska P: Thrombocytopenia in relation to tumor size in patients with hepatocellular carcinoma. Oncology 83: 339-345, 2012.

14. Carr BI, Guerra V, De Giorgio M, Fagiuoli S and Pancoska P Small hepatocellular carcinomas and thrombocytopenia. Oncology 83: 331-338, 2012.

15. Péus D, Newcomb N and Hofer S: Appraisal of the Karnofsky Performance Status and proposal of a simple algorithmic system for its evaluation. BMC Med Inform Decis Mak 13: 72, 2013.

16. Schwartz M, Roayaie S and Konstadoulakis M: Strategies for the management of hepatocellular carcinoma. Nat Clin Pract Oncol 4: 424-432, 2007.

17. Lencioni R and Llovet JM: Modified RECIST (mRECIST) assessment for hepatocellular carcinoma. Semin Liver Dis 30: $52-60,2010$

18. Li B, Yu J, Wang L, et al: Study of local three-dimensional conformal radiotherapy combined with transcatheter arterial chemoembolization for patients with stage III hepatocellular carcinoma. Am J Clin Oncol 26: e92-e99, 2003.

19. Cox JD, Stetz J and Pajak TF: Toxicity criteria of the Radiation Therapy Oncology Group (RTOG) and the European Organization for Research and Treatment of Cancer (EORTC). Int J Radiat Oncol Biol Phys 31: 1341-1346, 1995.

20. Yamashita H, Onishi H, Matsumoto Y, et al: Local effect of stereotactic body radiotherapy for primary and metastatic liver tumors in 130 Japanese patients. Radiat Oncol 9: 112, 2014.
21. Avila MA, Berasain C, Sangro B and Prieto J: New therapies for hepatocellular carcinoma. Oncogene 25: 3866-3884, 2006.

22. Peck-Radosavljevic M: Hepatocellular carcinoma: The place of new medical therapies. Therap Adv Gastroenterol 3: 259-267, 2010.

23. Lawrence TS, Robertson JM, Anscher MS, Jirtle RL, Ensminger WD and Fajardo LF: Hepatic toxicity resulting from cancer treatment. Int J Radiat Oncol Biol Phys 31: 1237-1248, 1995.

24. Purdy JA: 3D treatment planning and intensity-modulated radiation therapy. Oncology (Williston Park) 13: 155-168, 1999.

25. Park HC, Seong J, Han KH, Chon CY, Moon YM and Suh CO: Dose-response relationship in local radiotherapy for hepatocellular carcinoma. Int J Radiat Oncol Biol Phys 54: 150-155, 2002.

26. Yamada K, Izaki K, Sugimoto K, et al: Prospective trial of combined transcatheter arterial chemoembolization and three-dimensional conformal radiotherapy for portal vein tumor thrombus in patients with unresectable hepatocellular carcinoma. Int J Radiat Oncol Biol Phys 57: 113-119, 2003.

27. Yoon HI, Lee IJ, Han KH and Seong J: Improved oncologic outcomes with image-guided intensity-modulated radiation therapy using helical tomotherapy in locally advanced hepatocellular carcinoma. J Cancer Res Clin Oncol 140: 1595-1605, 2014

28. Chen D, Wang R, Meng X, et al: A comparison of liver protection among 3-D conformal radiotherapy, intensity-modulated radiotherapy and RapidArc for hepatocellular carcinoma. Radiat Oncol 9: 48, 2014.

29. Yao Q, Zheng R, Xie G, et al: Late-responding normal tissue cells benefit from high-precision radiotherapy with prolonged fraction delivery times via enhanced autophagy. Sci Rep 5: 9119, 2015.

30. Mornex F, Girard N, Beziat C, et al: Feasibility and efficacy of high-dose three-dimensional-conformal radiotherapy in cirrhotic patients with small-size hepatocellular carcinoma non-eligible for curative therapies - mature results of the French Phase II RTF-1 trial. Int J Radiat Oncol Biol Phys 66: 1152-1158, 2006.

31. Toramatsu C, Katoh N, Shimizu S, et al: What is the appropriate size criterion for proton radiotherapy for hepatocellular carcinoma? A dosimetric comparison of spot-scanning proton therapy versus intensity-modulated radiation therapy. Radiat Oncol 8: 48, 2013 